\title{
Objective evaluation of pain in various spinal diseases: neuropeptide immunoreactivity in the cerebrospinal fluid
}

\author{
Hiroshi Imasato $^{1}$, Kensei Nagata ${ }^{1}$, Sanshiro Hashimoto ${ }^{1}$, Hironori Komori ${ }^{2}$ and Akio Inoue ${ }^{1}$ \\ ${ }^{1}$ Department of Orthopaedic Surgery, ${ }^{2}$ Pediatrics and Child Health, Kurume University School of Medicine, Kurume \\ 830, Japan
}

A quantitative analysis was performed of substance P-like immunoreactivity (SPLI) and of $\beta$ endorphin-like immunoreactivity ( $\beta$-ENDLI), in the cerebrospinal fluid (CSF) in various diseases. The results reported to date have not been consistent. The purpose of this study was to investigate whether or not the concentration of SPLI or that of $\beta$-ENDLI in CSF demonstrated any potential for assessing the degree of subjective pain in various spinal diseases. SPLI in CSF was measured by radioimmunoassay in 158 patients with a spinal disease; involving 57 patients with a lumbar disc herniation (LDH), 38 with lumbar canal stenosis (LCS), 46 with cervical myelopathy (CM) and 17 with cervical radiculopathy (CR), and also in 20 healthy controls. $\beta$-ENDLI in CSF was measured in 25 of these same patients; involving 12 with LDH, seven with LCS and six with CM, and also five of the same controls. The concentration of serum SPLI was also measured in 50 of these 158 . The severity of pain was self-evaluated by each patient using a linear visual analogue scale (VAS). Their Japanese Orthopaedic Association (JOA) score was also calculated objectively using the clinical findings. Correlations were investigated among the concentrations of SPLI and $\beta$-ENDLI in the CSF and the VAS and JOA clinical assessments of these patients. The concentration of SPLI in CSF was significantly higher in various spinal diseases than in controls $(P<0.05)$, and was correlated with the severity on the VAS and with the JOA score. However, $\beta$-ENDLI was not correlated with either the VAS or the JOA score. We conclude that the measurement of the SPLI concentration in CSF has the potential for assessing objectively the severity of pain associated with various spinal diseases.

Keywords: substance P; $\beta$-endorphin; pain; lumbar disc herniation; lumbar canal stenosis; cervical myelopathy; cervical radiculopathy

\section{Introduction}

The activities of endogenous transmitters such as the transmitters of nociceptive information and the endogenous neuropeptides for pain reduction have been intensively investigated in recent years to clarify the pathway of pain sensation. Substance $\mathrm{P}$ (SP) discovered in the bovine GI tract by von Euler and Gaddum $^{1}$ in 1931 is a basophilic neuropeptide with 11 amino acids. $^{2}$ Immunohistochemical investigations have demonstrated that SP occurs in the terminal ends of primary afferent fibers in the spinal dorsal horn. ${ }^{3}$ Most neuropeptides including SP are present in the small cells of the spinal dorsal horn, and they exist preferentially in the axon of small fibers such as the myelinated $\mathrm{A} \delta$-fibers or unmyelinated $\mathrm{C}$-fibers in the primary afferent fibers. SP stimulates the nociceptive receptors in the spinal dorsal horn. Therefore, SP has been suggested to function as a transmitter of nociceptive information.

Correspondence: Dr H Imasato, MD
The highest levels of $\beta$-endorphin ( $\beta$-END) are demonstrated in the pituitary gland, and it has also been found in the other parts of the hypothalamus, in the gastrointestinal tract, the uterus and in the placenta. $\beta$-END is known to inhibit the release of the neurotransmitter-acetylcholine or noradrenaline with or without metenkephalin or dynorphin.

In diseases where there is pain, the level and distribution of these neurotransmitters in the cerebrospinal region are possibly different from conditions with no pain. While several quantitative analyses for SP and $\beta$-END in CSF in various diseases have been performed, no consistent results have so far been obtained. ${ }^{4}$ This has likely been because the grades of pain in the same disease varies naturally from time to time in any one patient, as well as from patient to patient. This has made any comparison difficult between the grade of pain and the concentration of SP or $\beta$-END. In the present study, we measured the concentrations of SP and $\beta$-END in CSF in various spinal diseases by radioimmunoassay, and compared 
these with the subjective severity of the clinical pain, together with objective findings, in various diseases, to evaluate whether or not SP or $\beta$-END in CSF demonstrated any potential for assessing the severity of pain experienced in these diseases.

\section{Materials and methods}

The concentration of SP-like immunoreactivity (SPLI) in CSF was measured using radioimmunoassay (RIA) in 158 cases of various spinal diseases, involving 57 patients with lumbar disc herniation (LDH), 38 with lumbar spinal canal stenosis (LCS), 46 with cervical myelopathy $(\mathrm{CM}), 17$ with cervical radiculopathy $(\mathrm{CR})$, and 12 with a lower limb fracture (FR). The controls were 12 patients with a lower limb healed fracture and no pain, and eight with lower limb benign soft-part tumor and no pain. The concentration of SPLI in the CSF of these 190 cases was measured using RIA. The concentration of serum SPLI was also measured in 50 of these 158 with various spinal diseases. We also assayed the concentration of $\beta$ END-like immunoreactivity ( $\beta$-ENDLI) in the CSF in 25 of these 158 with a spinal disease, involving 12 patients with LDH, seven with LCS and six with CM, and five of the controls. All patients gave written informed consent.

Samples of CSF (generally two specimens each $1.5 \mathrm{ml}$ ) were obtained by lumbar puncture from each patient when undergoing myelography or having lumbar anesthesia. The CSF was transferred to a test tube containing EDTA, mixed, and stored at $-80^{\circ} \mathrm{C}$. At the same time, serum was obtained and stored at $-80^{\circ} \mathrm{C}$. The concentration of the SPLI in the CSF was measured using the RIA technique using labeled $\mathrm{N}^{\alpha}$ hydroxyphenylpropinyl-substance $\mathrm{P}$ (Otsuka Assay Laboratories, Tokushima, Japan). $\beta$-END was also labeled with ${ }^{125} \mathrm{I}$, and the concentration of $\beta$-ENDLI was determined from RIA using the two-antibody method (PLASMA $\beta$-ENDORPHIN, Incstar Co. Osaka, Japan) (Table 1).

Pain was assessed by means of a visual analogue scale (VAS) consisting of a $100 \mathrm{~mm}$ horizontal line marked 'No pain' at its left end and 'Worst imaginable pain' at its right end. On repeated assessments, the patients were not shown their previous ratings. Low-back pain was also assessed by the Japanese Orthopaedic Association (JOA) score, which involved subjective symptoms, clinical signs, and restrictions in ADL.

Those data which were evaluated immediately before lumbar puncture were analyzed using Student's test and one way analysis of variance (ANOVA) (Table 2).

\section{Results}

Concentrations of SPLI in CSF and in serum

No significant relationships were found between SPLI and age or sex in the CSF of patients and in controls.
Table 1 Procedure of SP radioimmunoassay

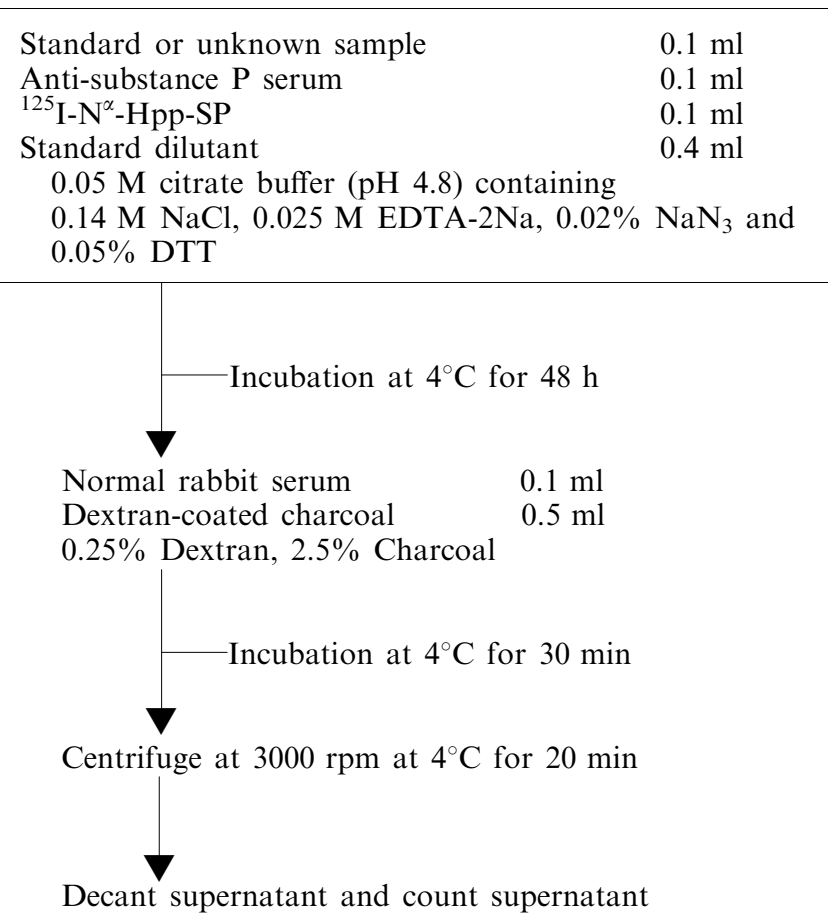

The endogenous concentration of SPLI in the CSF from 20 controls was $18.5 \pm 3.6 \mathrm{pg} / \mathrm{ml}$. The mean concentrations $(\mathrm{pg} / \mathrm{ml})$ of the SPLI in the CSF of patients are presented in Table 3. These were significantly higher than in the controls. The SPLI in FR was significantly higher than in the various spinal diseases (Table 3 ). The serum concentration of SPLI in the 50 cases with a spinal disease was significantly higher than in the CSF. However, no correlation was found between the concentration of SPLI in the CSF and that in the serum.

The correlation of the severity of pain and the concentration of SPLI in CSF

We classified patients into a severe pain group or a mild pain group according to their degree of pain by VAS, JOA subjective symptoms, clinical signs and their restriction in ADL. Those in the severe pain group had $50 \mathrm{~mm}$ or more in VAS, less than five points in JOA subjective symptoms, less than four points in clinical signs, and less than eight points in restriction in ADL, while those in the mild pain group had less than $50 \mathrm{~mm}$ in VAS, more than four points in JOA subjective symptoms, more than three points in clinical signs, and more than seven points in their restriction in $\mathrm{ADL}$.

There was a positive significant correlation between the SPLI level in the CSF and the VAS $(P<0.05)$ (Figure 1). The concentration of SPLI in the CSF was $36.6 \pm 4.0 \mathrm{pg} / \mathrm{ml}$ in the severe pain group as classified by VAS and was $26.0 \pm 3.3 \mathrm{pg} / \mathrm{ml}$ in the mild pain 
group as classified by VAS $(P<0.05)$. However, no significant difference was found between the SPLI of the mild pain group and that of the controls (Figure 2 ). The concentration of SPLI in the CSF in the severe pain group, and of the mild pain group, in those with a lumbar disease as classified by JOA subjective symptoms was $40.3 \pm 3.6 \mathrm{pg} / \mathrm{ml}$, and $25.4 \pm 4.7 \mathrm{pg} / \mathrm{ml}$, respectively $(P<0.05)$, and no significant difference also was found between the SPLI in the mild pain

Table 2 Assessment of treatment for low back pain: Japanese Orthopaedic Association

\section{Subjective Symptoms}

A. Low back pain

(9 points)

a. None

b. Occasional mild pain

c. Frequent mild or occasional severe pain

d. Frequent or continuous severe pain

B. Leg pain and/or tingling
a. None
b. Occasional slight symptom
c. Frequent slight or occasional severe
d. symptom

C. Gait

a. Normal

b. Able to walk farther than 500 meters although it results in pain, tingling and/or muscle weakness

c. Unable to walk farther than 500 meters owing to leg pain, tingling, and/or muscle weakness

d. Unable to walk farther than 100 meters owing to leg pain, tingling, and/or muscle weakness

II. Clinical Signs

A. Straight-leg-raising test

(6 points)

(including tight hamstrings)

a. Normal

b. $30-70$ degrees

c. Less than 30 degrees

B. Sensory disturbance
a. None
b. Slight disturbance (not subjective)
c. Marked disturbance

C. Motor disturbance (MMT)
a. Normal (Grade 5)
b. Slight weakness (Grade 4)
c. Marked weakness (Grades 3-0)

III. Restriction in ADL

(14 points)

\begin{tabular}{lccc}
\hline ADL & $\begin{array}{c}\text { Severe } \\
\text { restriction }\end{array}$ & $\begin{array}{c}\text { Moderate } \\
\text { restriction }\end{array}$ & $\begin{array}{c}\text { No } \\
\text { restriction }\end{array}$ \\
\hline a. Turn over while lying & 0 & 1 & 2 \\
b. Standing & 0 & 1 & 2 \\
c. Washing & 0 & 1 & 2 \\
d. Leaning forwards & 0 & 1 & 2 \\
e. Sitting (about 1 h) & 0 & 1 & 2 \\
f. Lifting or holding heavy & 0 & 1 & 2 \\
$\quad$ objects & 0 & 1 & 2 \\
g. Walking & & & \\
\hline
\end{tabular}

Table 3 Concentration of substance P-like immunoreactivity (SPLI) in the CSF of patients with various spinal diseases or a lower limb fracture and in controls

\begin{tabular}{lcc}
\hline Group & Number of cases & SPLI $(\mathrm{pg} / \mathrm{ml})$ \\
\hline Control & 20 & $18.5 \pm 3.6$ \\
LDH & 57 & $36.8 \pm 2.9^{*}$ \\
LCS & 38 & $43.3 \pm 3.4^{*}$ \\
CM & 46 & $33.9 \pm 3.1^{*}$ \\
CR & 17 & $35.1 \pm 5.2^{*}$ \\
FR & 12 & $57.7 \pm 5.5^{* *}$ \\
& & (mean \pm SE) \\
\hline
\end{tabular}

LDH: Lumbar disc herniation; LCS: Lumbar canal stenosis; CM: Cervical myelopathy; CR: Cervical radiculopathy; FR: Lower limb fracture $* P<0.05$ vs control; $* * P<0.05$ vs control, LDH, LCS, CM and CR

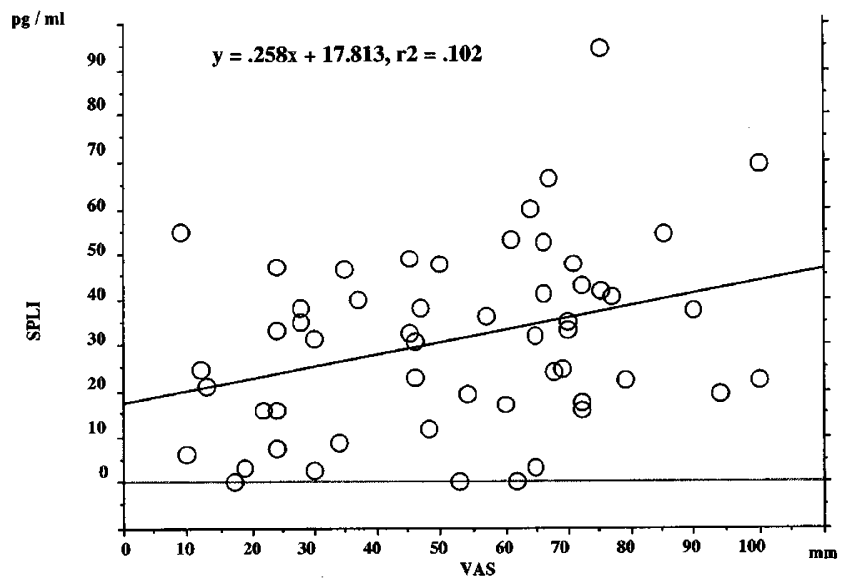

Figure 1 Correlation between SPLI in CSF and VAS in various spinal diseases. There was a positive significant correlation between the concentration of SPLI in CSF and VAS

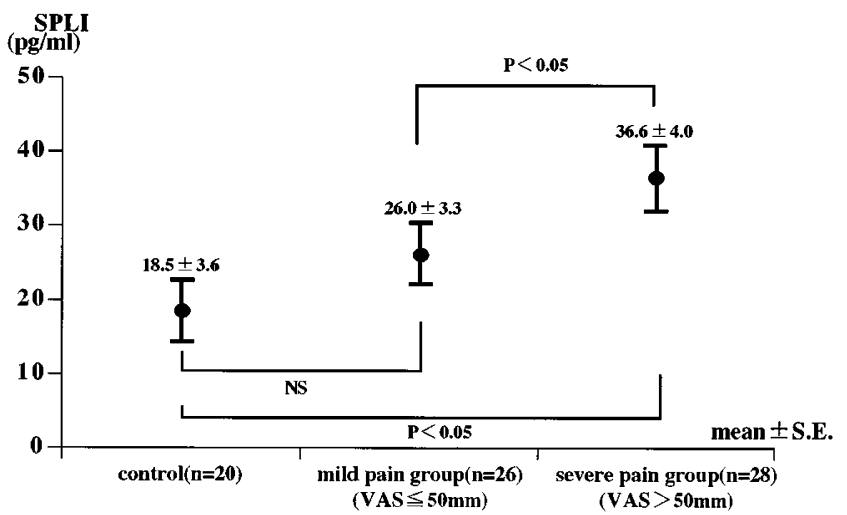

Figure 2 SPLI concentration in the mild pain group and in the severe pain group according to VAS in various spinal diseases 
group and in that of the controls. The SPLI level in the severe pain group $(32.5 \pm 5.7 \mathrm{pg} / \mathrm{ml})$ and that of the mild pain group $(35.3 \pm 4.0 \mathrm{pg} / \mathrm{ml})$ as classified by clinical signs were not significantly different. The SPLI level in the severe pain group $(41.3 \pm 5.1 \mathrm{pg} / \mathrm{ml})$ as classified by ADL was significantly higher than that of controls, whereas no significant difference was observed between the severe group and the mild pain group $(27.5 \pm 6.2 \mathrm{pg} / \mathrm{ml})$. The SPLI level in the positive cases by the SLR test was significantly higher than that of the controls $(P<0.05)$, but in proportion to the degree of the SLR result there was no significant difference. The SPLI in the 30 LCS cases with radicular pain $(48.5 \pm 3.3 \mathrm{pg} / \mathrm{ml})$ was significantly higher than that of the eight cases with no radicular pain $(23.9 \pm 7.9 \mathrm{pg} / \mathrm{ml})(P<0.01)$.

The relationship between the SPLI in CSF and the duration of the condition.

No correlation was found between the SPLI level in the CSF and the duration of the cervical or lumbar condition.

\section{$\beta$-ENDLI in the CSF}

In various spinal diseases, the level of SPLI was significantly higher than that in controls, whereas the level of $\beta$-ENDLI in lumbar spine diseases involving the LCS or LDH was significantly lower than that in $\mathrm{CM}$ and lower than that in controls (Table 4).

The correlation between the SPLI and the $\beta$-ENDLI in the $C S F$

There was no correlation between the level of SPLI and the level of $\beta$-ENDLI in the CSF in the 25 patients assayed at the same time.

The relationship of the severity of pain with $\beta$-ENDLI There was no significant correlation between the VAS score and the level of $\beta$-ENDLI, between the severe

Table 4 Concentration of $\beta$-endorphin-like immunoreactivity $(\beta$-ENDLI) in the CSF of patients with LDH, LCS, or $\mathrm{CM}$, and in controls

\begin{tabular}{lccc}
\hline Group & $\begin{array}{c}\text { Number of } \\
\text { cases }\end{array}$ & $\begin{array}{c}\text { SPLI } \\
(\mathrm{pmol} / \mathrm{l})\end{array}$ & $\begin{array}{c}\beta \text {-ENDLI } \\
(\mathrm{pmol} / \mathrm{l})\end{array}$ \\
\hline Control & 5 & $4.6 \pm 3.4$ & $9.6 \pm 0.7$ \\
LDH & 12 & $28.6 \pm 5.3^{*}$ & $4.9 \pm 1.0^{* *}$ \\
LCS & 7 & $34.7 \pm 12.7^{*}$ & $5.1 \pm 0.9^{* *}$ \\
CM & 6 & $37.8 \pm 4.8^{*}$ & $\begin{array}{c}8.6 \pm 0.8 \\
\text { (mean } \pm \mathrm{SE})\end{array}$ \\
\hline
\end{tabular}

LDH: Lumbar disc herniation; LCS: Lumbar spinal canal stenosis; CM: Cervical myelopathy; $* P<0.01$ vs control; $* * P<0.05$ vs control and CM pain group and the mild pain group as classified by either VAS or JOA score.

\section{Discussion}

Immunohistochemical investigations have demonstrated that SP and $\beta$-END were frequently seen in the small cells of the dorsal root ganglions and of the spinal dorsal horn, suggesting that these cells probably released SP. Because of the characteristic anatomical structure, deformation and any following compression in the nerve root would then release SP into the CSF. ${ }^{5}$ This was supported by the finding that the CSF level of SPLI was significantly higher in those patients with lumbar radiculopathy than those with no lumbar radiculopathy.

It is presumed that the $\beta$-END in CSF is released from the pituitary gland or from the brain, because the proopiomelanocortin which is a precursor of $\beta$-END is present only in the terminal end of the axon of the spinal cord and is not present in axon cells. Nilsson et $a l^{6}$ found no relationship between the SPLI concentration in plasma and that in CSF. The present study showed similar results, and we have concluded that SP in plasma is not likely transferred to the CSF.

The role of SP and $\beta$-END in the mechanism of pain: A pain stimulus applied to the extremity or the body trunk stimulates a peripheral receptor and is transmitted from the dorsal root to the spinal dorsal horn through primary afferent nerves. The stimulus then ascends through different synapses from the spinal dorsal horn to the supraspinal structures responsible for pain sensation via the spinothalamic or spinoreticular tract. The stimulus ascends along the reticular formation of the medulla oblongata to the somatic sensory cortex of the cerebrum via the thalamus. SP is known to be released in response to a pain stimulus as an excitatory transmitter of the nociceptive primary afferents. The transmission of nociceptive pain information through the spinal dorsal horn can be suppressed by various mechanisms. ${ }^{7}$ One mechanism involves the opioid peptides of the brain stem (especially the nucleus reticularis giantocellularis of the medulla oblogata) which when released from the terminal end of a nerve, than acts on the descending fibers of the noradrenaline or serotonin system in the spinal dorsal horn to suppress the transmission of the nociceptive pain information. The opioid peptides containing neurons in the spinal dorsal horn also inhibit directly the transmission of nociceptive pain information. One mechanism is that opiods in the spinal dorsal horn have an inhibitory action on the release of an excitatory transmitter from the terminal end of the presynaptic nerve fiber. ${ }^{8}$ Tseng et $a l^{9}$ demonstrated experimentally in rat that the release of methionineenkephalin was increased dosage- dependently by an intraventricular administration of $\beta$-END. They proposed that $\beta$-END activated the descending inhibitory tract. 
The SPLI level is less stable in the serum than in the $\mathrm{CSF}$, and it is not likely to be valuable for assessing pain since the neuropeptides in plasma are easily influenced by many exogenous factors including food intake, drugs and even the times and method of drawing blood specimens. In contrast, the SPLI in the CSF is presumed to reflect pain more accurately. If SP was the transmitter of pain and opioids were the inhibitor, then the production and distribution of these neuropeptides in the cerebrospinal neuron of the patients with continuous ache impulse may be different from those with no pain. Nutt et al. reported that SPLI decreased in the CSF of patients with peripheral neuropathy or autonomic dysfunction ${ }^{10}$ and Almay et $a l^{11}$ also reported that SPLI levels were lower in chronic pain patients, with either neurogenic or idiopathic pain syndromes, than in the healthy volunteers. Moreover Masaki and Cramer et al ${ }^{12,13}$ have reported that SPLI levels increased in those with a lumbar or sacral condition, polyneuropathy, or spinal cord condition. In the present study, we have found that SPLI in the CSF in various spinal diseases and in those with a lower limb fracture showed a significantly higher level than in controls. Also, the SPLI level in the severe pain group (as classified by VAS and JOA subjective symptoms) was significantly higher than in the mild pain group. This finding suggested that the subjective complaint of pain could be objectively evaluated by the SPLI level in the CSF. The SPLI level in the CSF also was useful for the clinical assessment of pain. Von Knorring et al ${ }^{14}$ reported the function of opioid peptides in the CSF in the descending inhibitory tract of patients with chronic pain, and described that the dynorphin levels in the CSF decreased in patients with neurogenic pain, and that the dynorphin levels were correlated with the threshold pain. Masaki reported that $\beta$-ENDLI levels in the CSF significantly increased in those with a lumbar or sacral condition, and that the SPLI level was also higher in these conditions than in controls. Our results showed that the SPLI level was significantly higher in those with LDH or LCS than in controls, but $\beta$-ENDLI levels were significantly decreased. Masaki reported that the concentration of $\beta$-END was significantly higher in those with $\mathrm{LH}$, LCS, or CM than in controls. But in our study, this concentration was significantly lower in those with LH or LCS than in controls and there was no significant difference between those with $\mathrm{CM}$ and the controls. Whether the descending tract inhibition by $\beta$-END was promoted or not by the discharge of SP in response to radicular pain in LDH and in LCS is not yet clear. This issue is to be investigated in the future. Senba ${ }^{15}$ reported a role for SP and enkephalin in the dorsal root ganglion and the spinal horn using a continuous ache impulse technique. Production of SP in the dorsal root ganglion increased by an ache impulse in the periphery and was transported to the spinal dorsal horn with increase in the discharge of SP and with increases in the opioid peptides (enkephalin and dynorphin). Whereas in patients with a continuous ache impulse, a large amount of SP produced in the spinal dorsal horn is considered to control the function of these opioid peptides. This suggested that the discharge of SP increased in the spinal dorsal horn and controlled the analgesic reaction by the opioid peptides. We conclude that although pain activates $\beta$ END inhibition in the descending tract, this pathway is restrained by the feed-back action of SP in patients with algesthesia who are sensitive to continuous ache impulses. In this study, the concentration of $\beta$-END in the CSF was not related with either VAS or the JOA score of clinical pain, and so was not regarded as useful for the clinical assessment of pain.

The findings from this study indicated that the concentration of SPLI in the CSF was useful for objectively evaluating pain caused by a spinal disease, especially lumbar radiculopathy while there was some limitation to such evaluation. One problem was the relationship between the SPLI concentration in the CSF and the VAS. A significant increase in SPLI occurred in severe cases (having a VAS exceeding $50 \mathrm{~mm}$ ) compared to mild cases on controls, with no significant difference between mild cases and controls. A significant increase in SPLI was also seen in severe cases according to the JOA score for lumbar radiculopathy and, again, no significant difference between mild cases and controls. Therefore, SPLI was concluded to be of little use for evaluating pain in mild cases. The SPLI concentrations were also significantly higher in patients with radicular symptoms and lumbar disease, and there appeared to be a close relationship between radiculopathy and SP release, but the SPLI level did not differ significantly from that in controls of patients without radicular symptoms.

In the evaluation of patients having a high VAS and other high pain scores but a low SPLI value, then it is first necessary to exclude feigned or psychogenic pain. It is likely that effects due to various types of mental stress can appear, especially in chronic patients. Alway et $a l^{11}$ reported that the SPLI level in the CSF was decreased in patients with chronic pain, especially in those with polyneuropathy. In our study, we did not find any close relationship between the duration of lumbar disease and the SPLI concentration, though there are possibly different mechanisms for the expression of acute and of chronic pain. Yokota ${ }^{16}$ noted a difference between the causes for acute and for chronic shooting pain related to intervertebral disk herniation. Acute pain was associated with dorsal root compression, but the characteristics of the compressed dorsal root nerve fibers changed in the chronic stage and the altered reaction to mechanical stimulation as well as spontaneous excitation of the spinal gangliocytes became involved in the expression of pain. Thus, there are limitations in only using the SPLI concentration for diagnosis of chronic pain, and other objective data as well as psychiatric assessment should be considered. 


\section{References}

1 von Euler U, Gaddum JH. An unidentified depressor substance in certain tissue extracts. $J$ Physiol 1931; 72: 74-87.

2 Chang MM, Leeman SE. Isolation of sialogenic peptide from bovine hypothalamic tissue and its characterisation as a substance P. J Bio Chem 1970; 245: 4784-4790.

3 Hökfelt $\mathrm{T}$, Elde R, Johansson $\mathrm{O}$ et al. Immunohistochemical evidence for separate populations of somatostatin-containing and substance P-containing primary afferent neurons in the rat. Neuroscience 1976; 1: $131-136$.

4 Harigaya Y. Somatostatin and substance P in the cerebrospinal fluid of some nuerodegenerative disorders. Kitakantou Med 1990; 40: 287 - 296 (in Japanese with English abstract).

5 Björn R, Mark DB, Göran L. Pathoanatomy and pathophysiology of nerve root compression. Spine 1984; 9: 7-13.

6 Nilsson G, Pernow B, Fisher GH, Folker K. Radioimmunological determinations of substance P. In $U$ von Euler and B Perrow (eds), Substance P. Raven Press: New York 1977, pp $41-48$.

7 Kuraishi Y et al. Noradrenergic inhibition of the release of substance $\mathrm{P}$ from the primary afferents in the rabbit spinal dorsal horn. Brain Research 1985; 359: $177-182$.

8 Inoki R. Endorphin and pain control. Pain Research 1991; 6: 1 -7 (in Japanese).

9 Tseng LF, King RC, Fujimoto JM. Release of immunoreactive met-enkephalin by intraventricular beta-endorphin in anesthetized rats. Regulatory Peptides 1986; 14: $181-192$.

10 Nutt JH et al. Substance $\mathrm{P}$ in human cerebrospinal fluid: reductions in peripheral neuropathy and autonomic dysfunction. Neurology 1980; 30: $1280-1285$.

11 Almay BGL et al. Substance $\mathrm{P}$ in CSF of patients with chronic pain syndromes. Pain 1988; 33: 3 -9.

12 Masaki K. A study of $\beta$-endorphin and substance $\mathbf{P}$ level in cerebrospinal fluid in lumbar lesions. Tokushima $J$ Exp Med 1987; 34: $61-69$.

13 Cramer $\mathrm{H}$ et al. Cerebrospinal fluid immunoreactive subtance $\mathrm{P}$ and somatostatin in neurological patients with peripheral and spinal cord disease. Neuropeptides 1988; 12: 119-124.

14 Von Knorring L, Almay BGL, Johansson F, Terenius L. Pain perception and endorphin levels in cerebrospinal fluid. Pain 1978; 5: $359-365$.

15 Senba E, Noguchi K. Neuropeptides in the pain transmission system: A morphological study. Pain Clinic 1989;10: 39 - 49 (in Japanese).

16 Yokota T. Transmission of pain sensation in spinal nerve system. In: Basic Mechanisms of Clinical Pain. Nankodo Co: Tokyo 1990, pp 20-22 (in Japanese). 